\title{
RF Ion Gun Injector in Support of Fusion Ship II Research and Development
}

\author{
G. H. Miley, Y. Shaban and Y. Yang \\ University of Illinois, U-C, Department of Nuclear, Plasma and Radiological Engineering \\ 103 S. Goodwin Ave., Urbana, IL 61801 USA \\ (217) 333-3772,ghmiley@uiuc.edu
}

\begin{abstract}
Ion injection into the Inertial Electrostatic Confinement (IEC) fusion power plant used in the design of the high performance Fusion Ship II (Burton et al., 2003) is a key technological issue for development of this concept. This paper discusses the design and initial experiments with a radiofrequency (RF) ion gun designed for this purpose. The RF Gun design described here was found to have some important advantages over other ion gun designs: simple construction, a higher extraction efficiency; $10\left(\left(\mathrm{~mA} / \mathrm{cm}^{2}\right) / \mathrm{W}\right)$, a lower divergence; $10.8 \pm 0.36 \mathrm{mrad}$, and a very intensive ion flux; $6 \times 10^{18}$ ions $/\left(\mathrm{cm}^{2} . \mathrm{sec}\right)$ measured at $0.27 \mathrm{~Pa}$.
\end{abstract}

\section{INTRODUCTION}

The inertial electrostatic confinement (IEC) power source employed in the very attractive Fusion Ship II design used neutral-beam injection for fueling and heating (Burton et al., 2003). Fusion Ship II was designed to achieve a fast 1year manned round trip mission to Jupiter. The present paper describes recent progress in IEC research using a newly designed external ion source, ILLIBS (Illinois Ion Beam Source) for ion injection into an IEC. This source uses a RF-generated plasma in an unique "index" magnetic-field design. These experiments represent a first step toward an ion-injected IEC such as envisioned for Fusion Ship II. Such operation allows beam formation and trapping in the IEC below the normal (Paschen curve) breakdown gas pressure, significantly reducing chargeexchange losses. The ILLIBS design provides a high ion current and high extraction efficiency. With ILLIBS, an injected deuterium ion current of $6 \times 10^{18}$ ions $/\left(\mathrm{cm}^{2}-\mathrm{sec}\right)$ was measured at $65 \mathrm{~mA}$ with a beam diameter of $2.89 \mathrm{~mm}$. A D-D fusion neutron rate of $2 \times 10^{7} \mathrm{n} / \mathrm{sec}$ was achieved with the IEC grid voltage and current at $75 \mathrm{kV}$ and $15 \mathrm{~mA}$ respectively, and a $0.16 \mathrm{~Pa}$ deuterium background pressure. The ILLIBS was operated at $100 \mathrm{Watt}$ RF power and 20 Watt dc magnetic field. This data is very encouraging and is in line with the theoretical model used in the conceptual design of the injected D-He ${ }^{3}$ IEC in Fusion Ship II. This ion injection technique, along with the development of a magnetically-channeled IEC array (Miley, 2004), represent two of the key physics issues involved in high power IEC development. The following sections provide a detailed description of the ILLIBS gun and its performance in ion injection experiments using a small IEC device.

\section{BACKGROUND - ION INJECTORS}

The use of ion beam sources and their applications span a broad spectrum of technologies. This includes ion beam applications in the fields of solid-state device fabrication, surface modification, treatment for tool wear resistance, thin film deposition, semiconductor ion implantation, fabrication of electronic devices, sheet metal processing surface analytical techniques, and ion-beam etching, just to name a few. This paper presents the results of a new technique to generate and extract a high current of energetic ion beam with minimum divergence. Ions are extracted from a plasma generated with Inductive Coupled Radiofrequency (ICRF ) waves interacting with the plasma in an appropriate magnetic field geometry. The main components of ILLIBS ion source device are described in the 
following sections. This particle unit was sized for use on a small (64 cm diameter) IEC device to provide a first test of the source and of IEC operation in the injected mode.

\section{Coaxial Helical Resonator Design}

The overall configuration for the ILLIBS source is shown in Figure 1. The main source of ionization is based on applying a RF wave through a coaxial copper helical resonator consisting of a single-layer coil within a copper shield. The helical coil (antenna) is made from magnet wire of wounding directly to the glass tube $(3.8 \mathrm{~cm}$ diameter x $25 \mathrm{~cm}$ length) with 1-mm clearance from the surface of the tubeand between turns. The RF signal at $13.5 \mathrm{MHz}$ is applied to the helical antenna, consisting of multiple turns of wire. The antenna occupies an area of $10 \mathrm{~cm} \times 3.8 \mathrm{~cm}$ while the length of the inner glass tube is $24 \mathrm{~cm}$. The copper shield is made from a 1-mm copper coating inside a stainless steel drum. A coaxial resonator is formed with an inner helical conductor placed within the copper shield. The helical resonator concept was suggested earlier by Macalpine and Schildknech (Macalpine and Schildknech, 1959). The present design falls with $\pm 10 \%$ of their approximate optimum design relations with several exceptions. The clearance between the helix and the shield was increased to avoid voltage flashover. A coaxial copper shield cavity (10-cm diameter x 12-cm length x $0.1-\mathrm{mm}$ thickness) was used here to enhance the coupling of the RF electromagnetic waves into the plasma tube.

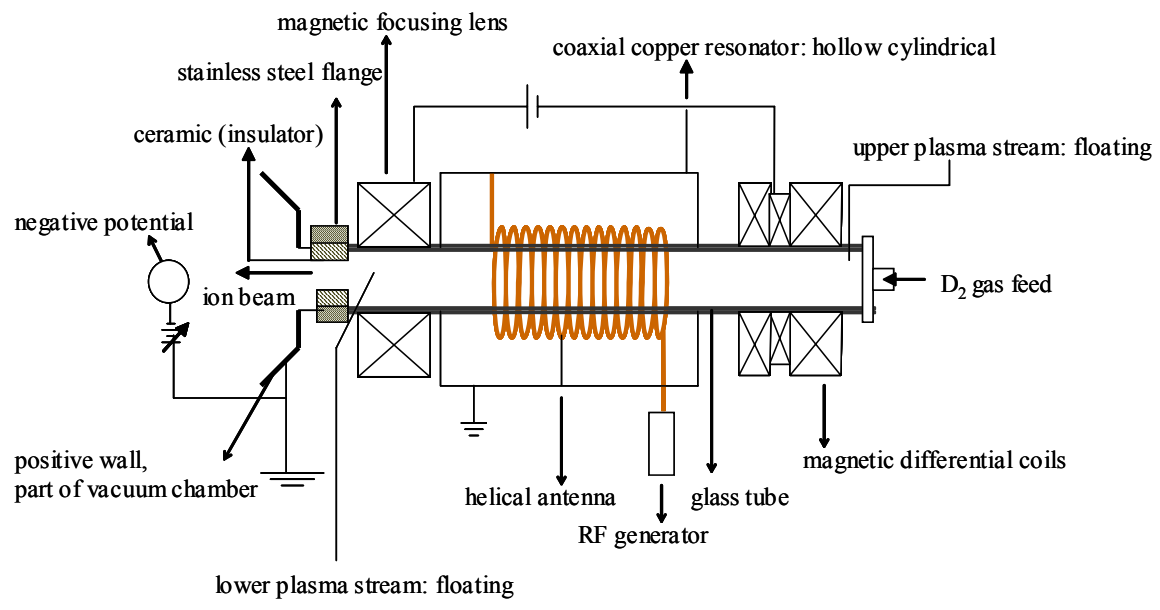

FIGURE 1. Typical Setup (Not to Scale) of the Illinois Ion Beam Source, ILLIBS (the main components shown are: magneticindex dc coils, coaxial cooper shielding, antenna, front magnetic focus coil, and floating exit nozzle)

\section{Differential Coils and Particle Trap}

The ILLIBS ion source is constructed such that it provides a charged-particle trap at the rear part of the source. The trap is based on applying a differential magnetic field generated by magnetic index coils, the resulting differential magnetic fields enhance the reflectivity, hence the confinement, of the ions generated by the RF field. In this case, three coils (joined in series) placed at the rear of the ion source were designed for that purpose. A dc current is employed in all coils. The first coil, furthest away from the antenna, was designed to initiate a magnetic field greater than the second coil. Thus the second coil acts as anti-reflection field for the first one, decreasing the ion reflectance. The third coil has a B-field greater than the second one but equal to the first one. Thus it serves as a reflector field to the second one, and so on. The reflectivity of ions, i.e. the particle trap efficiency, increases with the number of magnetic-index coils. However, to minimize the complexity of this preliminary design only 3 coils were used. The purpose of the added front coil was to focus and extract the deuterium atomic and molecular ions by reducing the ion orbital radius. This then effectively provides a magnetic extraction "nozzle". 
The aperture (floating nozzle) of the extraction opening was $1.5-\mathrm{cm}$ in diameter. The aperture ring is insulated by a coaxial ceramic within a stainless steel flange, so the aperture floats electrically with respect to the flange. As shown in Figure 2, the ILLIBS was attached to the spherical IEC vacuum chamber, biased negatively at the center grid with the vacuum wall grounded. The kinetic energy of the extracted ions is controlled by the geometric aspect ratio $a / \mathrm{D}$, where $a$ and $\mathrm{D}$ are the aperture radius and the extraction gap between the gun exit and the maximum potential point, respectively. The extraction potential $\mathrm{V}$ for constant current is proportional to the inverse of the aspect ratio, i.e. $\mathrm{V} \sim(\mathrm{D} / a)^{1.3}$. The aspect ratio for the present case, 0.028 , was purposely designed to be low by using a large extraction gap $\mathrm{D}=26-\mathrm{cm}$, compared to typical ion source designs.

\section{ION CURRENT MEASUREMENT SET UP}

The normal setup of the IEC vacuum chamber uses a small cathode grid (negative bias) located at the center of a metallic vacuum chamber (anode) which is grounded (Miley and Gu, 1999). To carry out ion current measurements with ILLIBS, a solid metal sphere was substituted for the grid as shown schematically in Figure 2.

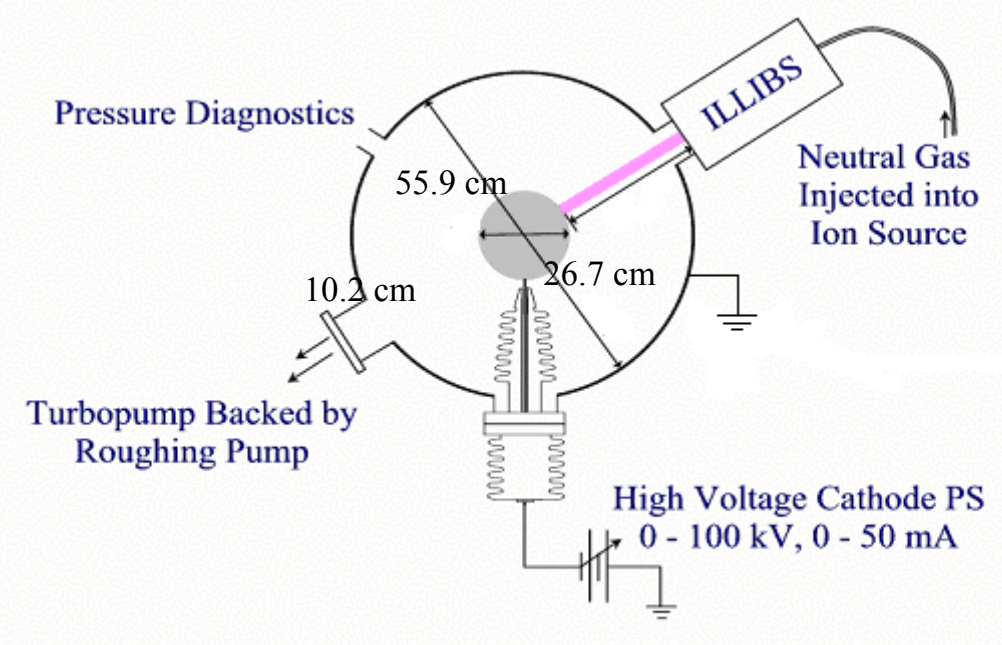

FIGURE 2. Schematic Representation of the Equipment Used in the Ion Current Measurements.

In Figure 2, the wall of the spherical chamber is grounded, while the negative target stainless steel ball at the center is at negative potential. The ion source ILLIBS is electronically floated in this case. One of the goals of the ion current measurements was to examine the current limits and extraction efficiency for ILLIBS operating with deuterium. For that purpose a stainless steel ball of $101.6 \mathrm{~mm}$ diameter (1-mm thickness) was placed at the center of the IEC vacuum chamber (55-cm diameter). This metal ball was attached to a high voltage de power supply (100 $\mathrm{kV}$ and $50 \mathrm{~mA}$ maximum). The ball was biased negatively while the wall chamber was grounded. All experiments, in the present setup, were performed below the breakdown regime to avoid contributions of background ion currents to those generated by the ILLIBS source. The mean distance from the gun extraction exit to the ball surface was $267 \mathrm{~mm}$. With the RF power was fixed at 100 Watts the total input power deposited in the gun $\left(283 \mathrm{~cm}^{3}\right.$, tube volume) was varied according to the magnetic field as shown in Table 1 . The ion extraction power efficiency is defined as the ion current density extracted per input power. To avoid excess current going to the ball due to ionization currents inside the chamber volume, the PV (pressure $\mathrm{x}$ voltage) point of the background gas was always kept well below the breakdown value. The breakdown PV point for this present setup was $26.7 \mathrm{kV}-\mathrm{Pa}$. The maximum PV point reached in the present experiments was $3.60 \mathrm{kV}-\mathrm{Pa}$.

TABLE 1. Total Input Powers Deposited at a Given Magnetic Field (The $100 \mathrm{~W}$ RF power is included).

\begin{tabular}{l|lllll}
\hline Total input power (We) & 121 & 184 & 289 & 436 & 625 \\
\hline Magnetic field (Tesla) & 0.015 & 0.031 & 0.045 & 0.06 & 0.075 \\
\hline
\end{tabular}




\section{DEUTERIUM ION CURRENT MEASUREMENTS}

The experimental results of the ion current measurements are shown in Figures 3. It should be emphasized that thermionic emission, which occurs when a metal is heated, does not make a significant contribution to the measured ion current. This was confirmed by noting the current meter still read zero when the ball was biased to negative voltages up to $49 \mathrm{kV}$ at a background pressure up to $0.27 \mathrm{~Pa}$.

As shown in Figure 3, at $0.05 \mathrm{~Pa}$ and at $0.03 \mathrm{Tesla}$, the ion current increases slowly with the increase of the extracted voltage, reaching a peak value of $17.5 \mathrm{~mA}$ at $5.5 \mathrm{kV}$. At that point the current dropped sharply to $1 \mathrm{~mA}$ current remained constant. This drop in current is attributed to the transition from below to above the plasma discharge breakdown voltage. The region of interest for injected IEC operation corresponds to that below breakdown, i.e. below this sharp drop off in current. In a similar fashion, the peak and minimum currents (after the sharp drop) measured at $0.045,0.06$, and 0.075 Tesla are as follows: 7.5, 0.8 mA; $15,1.8 \mathrm{~mA}$, and 25, $0.8 \mathrm{~mA}$ respectively. The corresponding extraction potentials at the transition point are: $5,4.5,3.5$, and $5.5 \mathrm{kV}$ respectively. In the region below breakdown, the ion beam current generally increases with increasing the magnetic field except for the abnormally high results at 0.03 Tesla. The corresponding differential ion energy distribution is very close to Gaussian. The energy spread value, $\Delta \mathrm{E}^{1 / 2}$, , (corresponding to $1 / 2 \Delta \mathrm{I}_{\mathrm{m}}$ full-width at half-maximum) contains $76 \%$ of the total beam current. The $\Delta \mathrm{E}_{1 / 2}$ values corresponding to $0.075,0.06,0.045$, and 0.03 Tesla are: $2,1.5,1.0$, and 2.5 $\mathrm{keV}$, respectively.

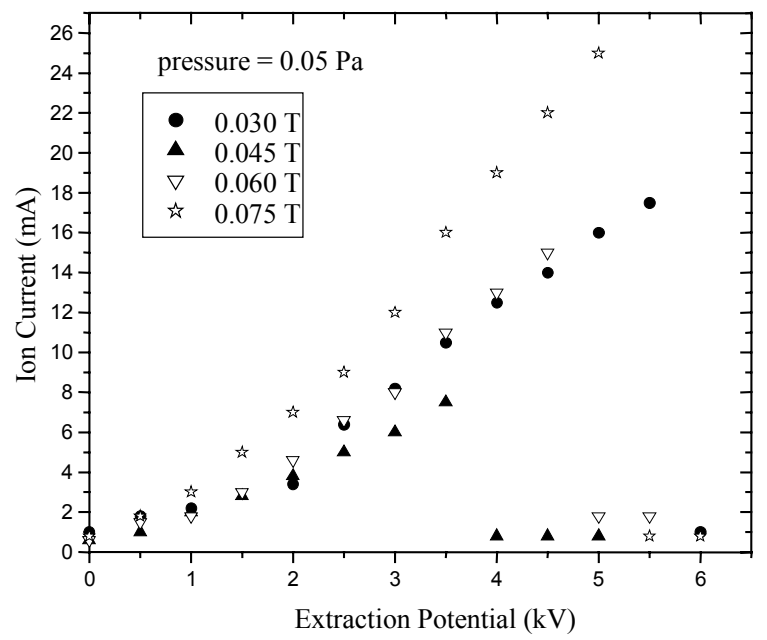

FIGURE 3. The Characteristic Behavior of the Ion Current Measured at Different Magnetic Fields.

\section{CURRENT AND BEAM DIAMETER MEASUREMENTS}

Figure 4 illustrates the ion current measurements at $0.27 \mathrm{~Pa}$. The maximum current was not obtained at this pressure due to the limitation of the power supply. At the point indicated in the figure, the ion beam drilled a $2.89 \pm 0.01 \mathrm{~mm}$ hole. The uncertainty of the measurements is $1 \mathrm{~mA}$ and 0 . At this pressure with a current $\sim 65 \mathrm{~mA}$ and extraction potential of 13.5 $\mathrm{kV}$, the ion beam started to melt a $6.6 \mathrm{~mm}^{2}$ spot in the stainless steel ball. The hole that was made by the ion beam is either equal to the diameter of the ion beam or slightly greater than the beam width because of expansion of the metal around the hot spot. Based on the melted spot, the effective ion beam diameter was determined to be $2.89 \pm$ $0.01 \mathrm{~mm}$. The current at which the hole occurred was $\sim 65 \mathrm{~mA}$ with an area and volume of $0.065 \mathrm{~cm}^{2}$ and 0.0065 $\mathrm{cm}^{3}$ respectively (the thickness of the ball is $\sim 1 \mathrm{~mm}$ ), while the power density of the ion beam was $13.5 \mathrm{~kW} / \mathrm{cm}^{2}$.

To obtain melting time data, the original video was converted to an AVI (audio video interleave) file accurate to \pm 0.03 seconds. Based on that file, the melt down of the stainless steel ball was estimated to occur in $60 \pm 3 \mathrm{msec}$. 
The corresponding energy density on the spot, based on the beam parameters and spot size, was $810 \mathrm{~J} / \mathrm{cm}^{2}$. The critical energy density (Incropera and Dewitt, 1996) required to melt stainless steel is $8.1 \mathrm{~kJ} / \mathrm{cm}^{3}$, and the energy density required to penetrate and melt a $1-\mathrm{mm}$ thick ball of the above properties is $808 \mathrm{~J} / \mathrm{cm}^{2}$. Using these values the melting rate of stainless steel is found to be $\sim 58 \mathrm{msec}$ in good agreement with the video observation.

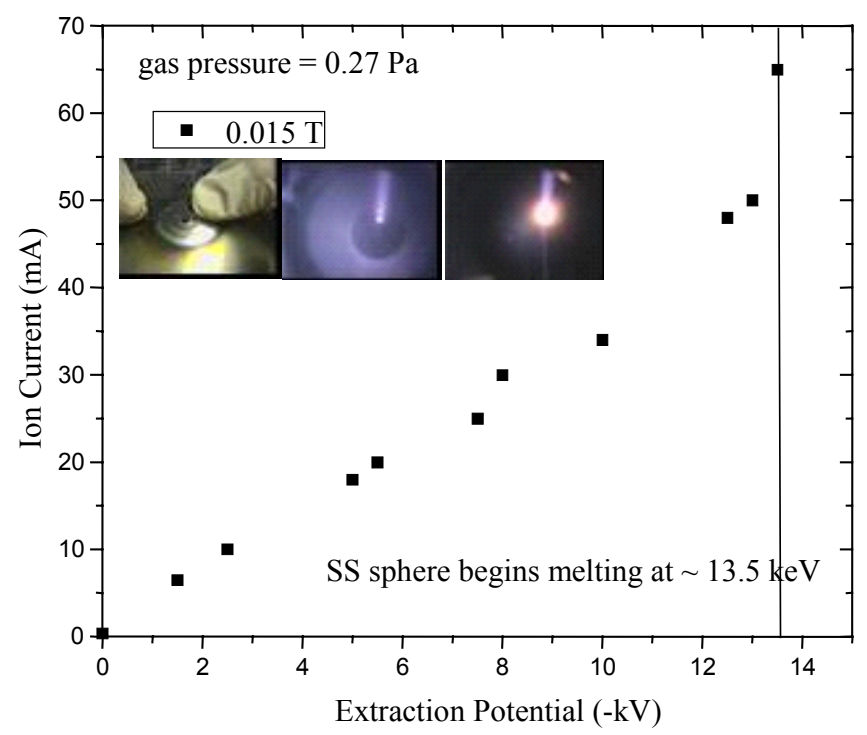

FIGURE 4. Variation of the Ion Beam Current with Extraction Potential at $0.27 \mathrm{~Pa}$.

\section{RELATIVE ION BEAM DIAMETERS AND DIVERGENCE MEASUREMENTS}

The relative beam diameters at pressures less than $0.27 \mathrm{~Pa}$ were measured optically using the projected beam spot from a video camera referenced to the size of the ball. The result using this technique at 2 mTorr and the actual melted hole size measured on the ball at 2 mTorr were in good agreement. To obtain the beam divergence, the far field angle law, $\theta=\mathrm{d} / \mathrm{D}$, is employed where $\mathrm{d}$ is the beam diameter measured at distance $\mathrm{D}(26.7 \mathrm{~cm})$ from the exit of the ion source. Thus beam divergence increases with the increase of pressure, and it is inversely proportional to the magnetic field. Measured Divergence values are shown in Figure 5. Data were taken at different pressures $(0.05$ $\mathrm{Pa}$ to $0.16 \mathrm{~Pa})$, and at different magnetic fields $(0.015$ Tesla to 0.075 Tesla). As expected the divergence is very small, decreasing with reduced background pressure (reduced scattering) and with increasing RF Gun Magnetic field (small "source" diameter). This data ca also be used to estimate the beam perveance.

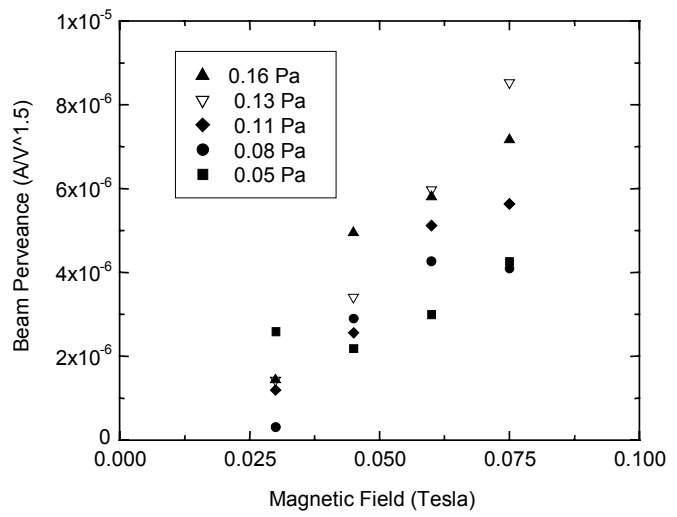

FIGURE 5. The Relative Effective Ion Beam Divergence at $267 \mathrm{~mm}$ for Various Pressures and Magnetic Fields. 
The equivalent ion beam perveance $\mathrm{P}, \mathrm{P}=\left(\frac{m_{i}}{m_{e}}\right)^{1 / 2} \frac{I}{V^{3 / 2}}$, is an important characteristic parameter for ion sources.

For the present ILLIBS P was found to be greater than $\sim 1 \times 10^{-6} \mathrm{~A} / \mathrm{V}^{3 / 2}$ (Figure 6). The ratio of the extraction gap $(267 \mathrm{~mm})$ to the aperture diameter $(15 \mathrm{~mm})$, was 17.8 in these measurements. The measured beam perveance is excellent compared to other ion beam sources. As expected it increased with RF gun magnetic field and with increasing pressure. There is an anomalous cross over in data for increasing pressure, possibly due to experimental uncertainties.

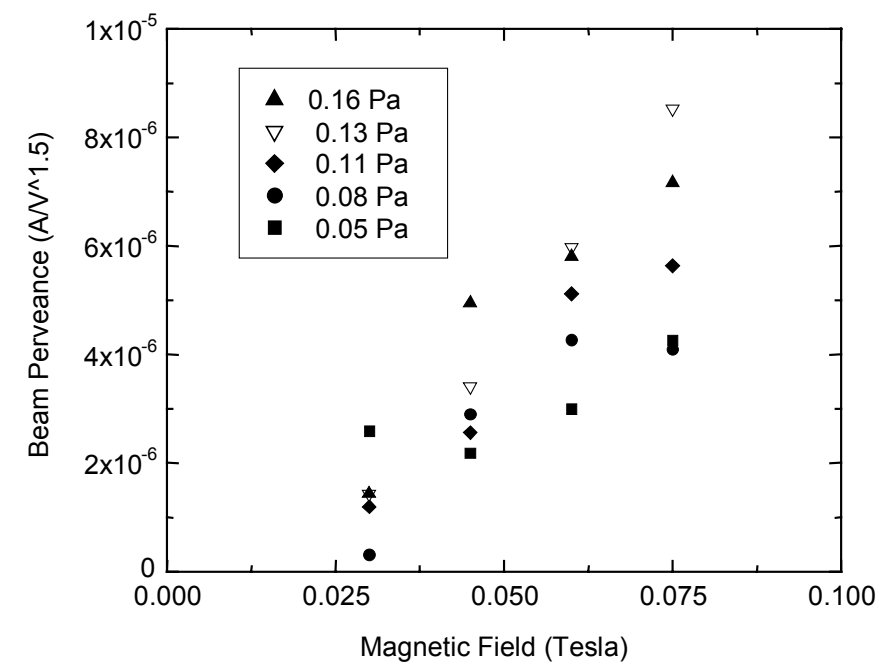

FIGURE 6. The Dependence of the Ion Beam Perveance on Magnetic Field for various with Gas Pressures.

\section{DISCUSSION AND CONCLUSION}

The next step needed in the development of a high power IEC system such as envisioned in Fusion Ship II involves demonstration of a suitable ion injection technique. In this paper we had described the design and initial experiments for a new type of magnetic-index type RF ion source (ILLIBS) that appears to be well suited for ion injection into the IEC. The experiments reported here used a small low current unit. Still the results are very encouraging, suggesting extrapolation to a high current device should be possible. To provide some feeling for the performance of this new ion source we compared its key characteristics to a wide variety of other types of ion sources, based on four key articles (Fitch, 1971; Lobanov, 1994; Brown, 1992; Takahashi, 1996). It was found that the magnetic-index approach in the ILLIBS configuration exhibits several advantages over other ion gun designs: simpler design, higher extraction efficiency; $10\left(\left(\mathrm{~mA} / \mathrm{cm}^{2}\right) / \mathrm{W}\right)$, lower divergence; $10.8 \pm 0.36 \mathrm{mrad}$, and a very intensive ion flux; $6 \times 10^{18}$ ions $/\left(\mathrm{cm}^{2} . \mathrm{sec}\right)$ measured at $0.27 \mathrm{~Pa}$. The corresponding ion current density at $0.27 \mathrm{~Pa}$ is greater than $1.0 \mathrm{~A} / \mathrm{cm}^{2}$ at an extraction potential of $13.5 \mathrm{keV}$. The next step in the evaluation of this injection technique would involve extending the present source design to higher currents and adding multiple sources to the experimental IEC. For symmetry, six injectors would be an ideal next step configuration. Indeed, the original ion gun experiments on an IEC performed by Hirsch (Hirsch, 1967) used six guns. These early guns suffered control problems and low ion currents, but even so gave impressive results. Experiments with guns such as ILLIBS should greatly extended the capability of IEC operation.

\section{ACKNOWLEDGMENTS}

This work is supported by NASA grant: NASA NPL SRA00-239. The authors would like to thank Professor Gary Eden, for his generous support of materials necessary for the experiments. Also, we deeply thank Professors David 
Ruzic, Hiromu Momota and Mark Kushner for their discussions and advice, and Dr. J.P.Allian for his helpful comments.

\section{REFERENCES}

Burton, R., Momota, H., Richardson, N., Shaban, Y., and Miley, G.H., "Fusion Ship II- A Fast Manned Interplanetary Space Vehicle Using Inertial Electrostatic Fusion," Space Technology and Applications International Forum (STAIF-2003), edited by M.S. El-Genk, American Institute of Physics, Conference Proceedings 654, 2003, pp. 553 - 562.

Brown, I.G., Dickinson, M.R., Galvin, J.E., and MacGill, R.A., "Development of a dc, broad beam MeV ion source," Rev. Sci. Instrument, 63(4), April 1992.

Fitch, R.K. and Rushton, G.J., “Low-Pressure Ion Source,” Journal of Vacuum Science and Technology, 379, (1971).

Hirsch, R.L., "Inertial Electrostatic Confinement of Ionized Fusion Gases," J. of Applied Physics, Volume 38, Number 11, October 1967.

Incropera, F.P. and Dewitt, D.P., Fundamentals of Heat and Mass Transfer, John Wiley \& Sons, N.Y., 1996

Lobanov, N.R., "The plasma-emitter type ion source," Rev Sci Instrum, (1994) 1399.

Macalpine, W.W., and Schildknech, R.O., "Coaxial Resonators With Helical Inner Conductors," Proceedings 1958 IRE National Convention, N.Y.C., NY, pp. 2099-2105, July 15, 1959.

Miley G.H., and Gu, Y., "IEC Neutron Source Development and Potential Well Measurements," Current Trends in International Fusion Research - Proceedings of the Second Symposium, Ottawa, ON, Canada, 1999.

Miley, G.H., Stubbers, R., Webber, J., Momota, H., "Magnetically-Channeled SIEC Array (MCSA) Fusion Device for Interplanetary Missions," in these proceedings of Space Technology and Applications International Forum (STAIF-2004), edited by M.S. El-Genk, American Institute of Physics, 2004.

Takahashi, T., Tomita, Y., Momota, H., and Shabrov, N., "Collisionless pitch angle scattering of plasma ions at the edge region of a field-reversed configuration," Plasma Physics, 4(12), 1997, pp. 4301-4308. 